\title{
Non-Liouvillean ion injection via resonantly enhanced two-photon ionization
}

\author{
B. A. Knyazev* \\ Novosibirsk State University, 630090 Novosibirsk, Russia \\ and Budker Institute of Nuclear Physics, 630090 Novosibirsk, Russia
}

(Received 12 November 2003; published 18 March 2004)

\begin{abstract}
The charge-exchange method is now one of the main techniques for ion injection into accelerators and storage rings. The disadvantages of conventional methods, based on the atom or ion stripping in a material target, are emittance growth, energy straggling, and production of ions in many charge states. Recently suggested stripping methods based on direct photoionization require employment of hard-UV lasers, which still do not exist and must obviously be very bulky and expensive. An alternative method, suggested for injection of proton beams, employs excitation of the atom to $3 p$ intermediate state with subsequent Lorentz ionization in a magnetic field gradient. This technique applies rigid requirements to laser characteristic and is not free of growing of the beam divergence. In this paper a variant of the stripping technique based on the resonantly enhanced two-photon ionization (RETPI) is considered. The technique allows ionization of singly charged ions of the elements from helium to bismuth. A variant of the technique can be used for proton injection. RETPI can be applied for both ion injection and stacking, as well as for diagnostics of ion beam characteristics on the orbit. Stripping efficiency can be about $100 \%$ for the singly charged ions having the singlet ground state and decreases for the other ions. Special methods for "cleaning" unwanted atomic states in such ions, that can provide high stripping efficiency, are discussed. Excimer lasers with very moderate parameters can be employed for implementation of this technique for almost all elements. Numerical examples show that for most of the singly charged ions and for hydrogen atom necessary laser-beam energy density is merely $0.5-8 \mathrm{~J} / \mathrm{cm}^{2}$ for a $1 \mathrm{~m}$ interaction region, and is 10 times higher for several light ions.
\end{abstract}

DOI: 10.1103/PhysRevSTAB.7.033501

PACS numbers: 29.27. Ac, 32.80.Fb

\section{INTRODUCTION}

The charge-exchange, or stripping, method of ion injection into accelerators and storage rings has essential advantages compared to the other conventional methods because this technique has no restrictions imposed on the storage ions on orbit by Liouville's theorem. Dempster in 1932 [1] obtained protons with a double energy of $45 \mathrm{keV}$ in a tandem "ground-22.5 keV-ground" accelerator using the charge exchange of protons in a hydrogen target. Double stripping of $\mathrm{H}^{-}$ions in a thin foil was first proposed by Alvarez [2] in 1951 for the tandem proton accelerators. He suggested the reconstruction of existing Van de Graaff accelerators to a tandem configuration, placing a $180^{\circ}$ magnet inside a high-voltage conductor. In the same paper he suggested also the application of the charge-exchange method for the tracing of orbits in the proton synchrotrons. However, he did not expect the application of this technique as a basic injection method because of a lack at that time of intense negative hydrogen ion sources.

Impressive development of the negative ion sources (see [3]) allowed in the following years obtaining high negative hydrogen ion currents. First experiments on the charge-exchange injection into a small storage ring using a gas target with a beam of accelerated $\mathrm{H}^{-}$ions have been carried out at the Institute of Nuclear Physics

*Electronic address: knyazev@phys.nsu.ru
(Novosibirsk) in the early 1960s [4,5]. A detailed analysis of the charge-exchange injection for the proton accelerators was performed by Dimov [6]. Later development of this technique is described in the review [7]. Nowadays the charge-exchange injection is a preferred injection method for proton machines. As an example one can mention the light ion storage ring COSY [8].

Since a hydrogen atom has only one electron, the charge-exchange injection of protons can be realized practically only by means of formation and acceleration of the negative hydrogen ions. The use of positive molecular ions, such as $\mathrm{H}_{2}^{+}$or $\mathrm{H}_{3}^{+}$, as primary particles is possible [9], but this technique has some disadvantages and is not applied commonly in practice [7]. For heavier elements there are more variants for the application of the ion stripping in the accelerator technique. The stripping in the material targets is already applied for multiple ionization of the accelerated low-charged ions and/or the injection of them into storage rings. This technique is used, for example, at Nuclotron (Dubna) [10], SIS and ESR (Darmshtadt) [11], and TSR (Heidelberg) [12].

Charge-exchange injection is a subject of special interest for the projects of heavy-ion driven inertial confinement fusion (HIF) [13-15]. There are several schemes for the HIF. In the project ITEP-TWAC $[13,16]$ multiplecharged ions are considered to be accelerated and directed to a D-T pellet. In American and European projects [17-19] to reduce space charge effects the acceleration of low-charged ions is considered. In both cases, 
however, to provide a necessary power density on the D-T pellet the ion beams have to be additionally compressed before aiming at the pellet. This assumes multiturn beam injection and bunch stacking, which can be performed by the charge-exchange technique.

The advantages and disadvantages of the chargeexchange injection of the heavy ions have been recently analyzed in detail by Dinev [20]. There are several problems in the standard charge-exchange scheme when one uses a material target on the orbit. First, the ions lose energy in the stripping foil due to atomic excitation and ionization that increases the energy straggling of the beam. Second, the Coulomb elastic scattering causes a change in the ion trajectory slope and leads to transverse emittance growth. In the case of the multiturn injection the four-dimensional transverse phase volume increases proportionally to the number of injection turns. The other important disadvantage, which does not exist in the case of proton machines, is a charge distribution function $F(q)$ for the ions passing through the target. This leads to the successive cut by the accelerator of all ions in charge states different from the equilibrium state charge and substantial losses of the accelerated particles.

A non-Liouvillean injection technique based on ion stripping via photoionization by hard vacuum ultraviolet (VUV) radiation has been recently proposed [21]. This technique promises to overcome the above mentioned disadvantages of the standard charge-exchange schemes but requires development of an intense VUV source. Two variants of an alternative scheme employing the resonance saturation of an intermediate level with following Lorentz stripping were suggested for ionization of fast hydrogen atoms $[22,23]$. This scheme will be discussed in Sec. VII.

In this paper I describe in more detail an injection method based on resonantly enhanced two-photon ionization (RETPI). This technique was first mentioned at the HIF-2002 Symposium (see [24]) and briefly described at a recent conference [25]. For many ions this injection technique can be implemented with commercially available excimer lasers. In the following sections of the paper we briefly review the existing projects of the photoionization stripping technique, describe principles of the novel technique, and analyze its potential capabilities.

\section{ION STRIPPING VIA DIRECT VUV PHOTOIONIZATION}

The application of intense radiation for ion beam injection had first been proposed by Arnold et al. [26] in 1977. They considered storing the atomic ions $\mathrm{I}^{+}$by direct or stepwise photodissociating the molecular ions $\mathrm{HI}^{+}$, having been previously accelerated, at injection into the storage ring. In the second case photodissociation of the molecular ion has to be carried out through an excited molecular state by xenon flashlamp or ruby laser radia- tion. The excitation energy of the molecular band is equal approximately to $3.6 \mathrm{eV}$, and to match the ruby laser photon energy $(1.78 \mathrm{eV})$ to this value the laser beam has to collide with the molecular beam being accelerated to $\beta=0.6$.

Direct photoionization of singly charged heavy ions

$$
X^{+}+\hbar \omega_{\mathrm{di}} \rightarrow X^{++}+e^{-}
$$

had been proposed by Rubbia [21] for injection of ions from linac into a storage ring. Hofmann $[17,27]$ suggested application of this technique for the final stage of pulse compression in the heavy-ion fusion reactor systems. Both schemes suppose employing a VUV laser for direct photoionization of the ions and the capture of the doublecharged ions produced on the equilibrium orbit, Fig. 1(a). One of the great advantages of this method in comparison with the foil stripping is its absolute selectivity. Since the next ionization potential is more than the photon energy, the radiation interacts only with the primary ions and the ions produced are not further disturbed being circulated on the same orbit.

The ionization potentials of singly charged ions lie between $10.0 \mathrm{eV}\left(\mathrm{Ba}^{+}\right)$and $75.6 \mathrm{eV}\left(\mathrm{Li}^{+}\right)$. The cross sections for direct photoionization near a threshold are as low as $10^{-17}-10^{-18} \mathrm{~cm}^{2}$ and an intense flux of hard radiation is required for efficient ion stripping. To decrease required light intensity Hofmann considered ion stripping via the excitation of an ion autoionization state [see Fig. 1(b)] that can be symbolically written for an ion $X^{+}$as

$$
X^{+}+\hbar \omega_{\mathrm{ai}} \rightleftharpoons X^{+* *} \rightarrow X^{++}+e^{-},
$$

where $X^{+* *}$ denotes the autoionization state. An autoionization cross section can reach a value of the order of $10^{-15} \mathrm{~cm}^{2}$ within narrow $(\sim 0.1 \mathrm{eV})$ peaks [28], which lies, however, at much higher photon energies than the direct ionization threshold. For example, for $\mathrm{Ba}^{+}$three

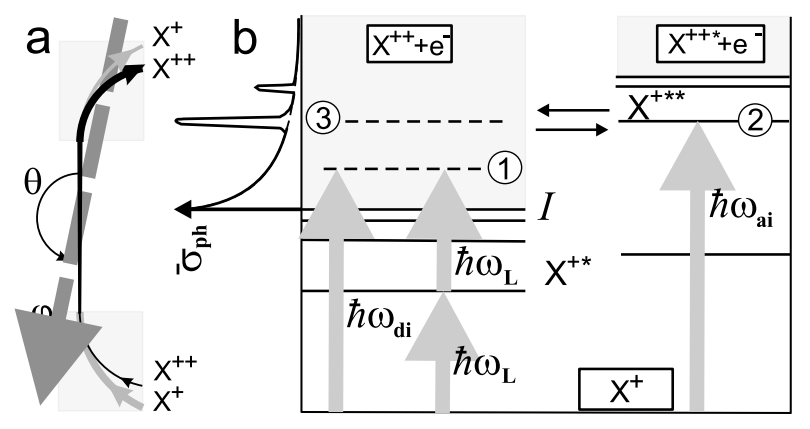

FIG. 1. Ion stripping via photoionization techniques: (a) schematics of photoionization; (b) Grotrian diagrams for direct photoionization (photon energy $\hbar \omega_{\mathrm{di}}$ ), photoionization through an autoionization state $\left(\hbar \omega_{\mathrm{ai}}\right)$, and resonantly enhanced photoionization $\left(\hbar \omega_{L}\right) ; 1$ and 3: states in continuum; 2: autoionization state; $\sigma_{\mathrm{ph}}$ : typical cross section of photoionization. 
autoionization peaks with $\sigma=(2.4-2.8) \times 10^{-15} \mathrm{~cm}^{2}$ lie at $21.2,26.9$, and $32.2 \mathrm{eV}$. According to [17] a similar cross section can be expected for $\mathrm{Bi}^{+}$at $25 \mathrm{eV}$.

Thus stripping the ions during time of flight of the bunch in a straight interaction region with the length of 1-10 $\mathrm{m}$ requires using a hard VUV light beam with low emittance, a short pulse length, and high intensity. Since in this spectral range there are no conventional laser sources with required parameters, authors of the papers $[17,21,27]$ proposed using for stripping a free electron laser (FEL).

There are two main problems in the implementation of this technique. First, FELs with necessary characteristics do not still exist though some of the existing FELs already generate radiation in VUV [29] and can, in principle, reach required parameters. Second, focusing and reflecting optical elements are obviously necessary for guiding of the light beams especially in the multipass optical systems. The last may be required to ensure multiple interaction of the light beam with the ion beam or beams. Unfortunately, there is a lack of suitable mirrors for the hard VUV radiation. Thus development of an alternative low-cost photoionization technique using soft VUV radiation is very desirable.

\section{ION STRIPPING VIA RESONANTLY ENHANCED UV PHOTOIONIZATION}

One can suggest two variants of ion ionization by low energy photons. The first is a direct multiphoton ionization (see, i.e., [30]). Since a cross section of the multiphoton ionization is very low, extremely high laser intensity is required. Resonantly enhanced two-photon ionization [31], Fig. 1(b),

$$
\begin{gathered}
X^{+}+\hbar \omega_{L} \rightleftharpoons X^{+*}, \\
X^{+*}+\hbar \omega_{L} \rightarrow X^{++}+e^{-}
\end{gathered}
$$

allows one to do it with substantially lower laser intensity. It retains the advantage of selectivity of the interaction only with the singly charged ions, but admits employment of more long-wave radiation in comparison to the case of the direct photoionization.

RETPI is a two-step process. On the first stage a singly charged ion is excited by intense resonance radiation to an intermediate level and then ionized by the next photon. Obviously, one or two lasers have to be employed to implement this technique. Selection of the laser depends on many factors: necessary wavelength, spectral bandwidth, total pulse energy, pulse length, beam divergence, and robustness. For distinctness, we assume further the excimer lasers as radiation sources. Commercially available ultraviolet excimer lasers have an extremely high service life [32]. They generate 1-10 J pulses at the repetition rate up to several $\mathrm{kHz}$. Characteristic laser pulse length is usually $10-40 \mathrm{~ns}$, but it can be extended to $100-300 \mathrm{~ns}$.

The ionization potentials $I$ of the singly charged ions lie between $75.6 \mathrm{eV}\left(\mathrm{Li}^{+}\right)$and $10.0 \mathrm{eV}\left(\mathrm{Ba}^{+}\right)$. To be photoionized the intermediate level $X^{+*}$ should lie not lower than the energy $I / 2$. Photon energies of widely used $\mathrm{KrF}$ and $\mathrm{ArF}$ lasers are equal to 4.99 and $6.41 \mathrm{eV}$, respectively. In the frame of a singly charged ion, laser radiation frequency $\omega_{L 0}$ transforms to

$$
\omega_{L}=\gamma(1-\beta \cos \theta) \omega_{L 0},
$$

where $\theta$ is the angle in the laboratory frame [Fig. 1(a)] between ion velocity and laser-beam direction, and $\gamma$ and $\beta$ are well-known relativistic variables.

We will assume below $\theta=\pi$. In this case

$$
\omega_{L}=\sqrt{\frac{1+\beta}{1-\beta}} \omega_{L 0} .
$$

Corresponding wavelengths of $\mathrm{KrF}$ and ArF lasers in the moving frame for the ions from $\mathrm{He}$ to $\mathrm{Bi}$ are given in Figs. $2-4$ for four values of the ion energy: $T=0.3,1.0$, 4.0 , and $10 \mathrm{GeV}$. Thin broken lines in the figures show the wavelengths corresponding to the energy $I / 2=\hbar \omega_{L}$ for the singly charged ion of each element. If the ion of an element has one or several resonance transitions with the wavelengths

$$
\lambda \leq \frac{4 \pi \hbar c}{I}
$$

lying lower than this line, it can be stripped to the next

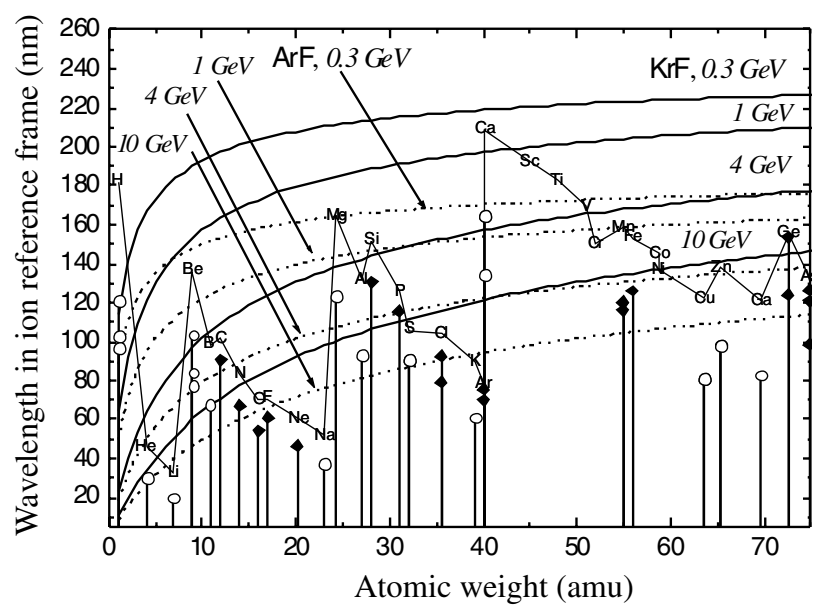

FIG. 2. Wavelengths of the excimer lasers in the moving frame for the singly charged ions of the first 75 elements. The points correspond to the resonance transitions for the singly ionized ions of the elements. The open circles correspond to the transitions with a singlet ground state; the diamonds denote the transitions with a multiplet ground state (the points for hydrogen are given for a neutral atom). A thin broken line marks the wavelengths corresponding to energy $I / 2$ for each ion. 


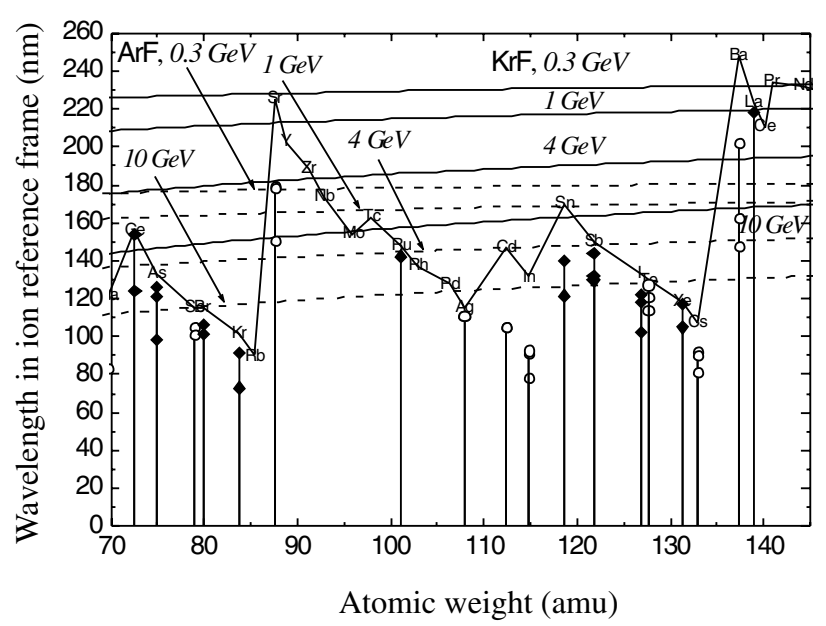

FIG. 3. Wavelengths of the excimer lasers in the moving frame for the singly charged ions of the intermediate mass elements. The legend is the same as in Fig. 2.

ionization state through one of these intermediate excited levels. Selected resonance transitions satisfying this requirement are also shown in the figures. For example, strontium ion $\mathrm{Sr}^{+}$, as it is seen from Fig. 3, has at least two transitions which lie lower than the limit. It can be ionized with the $\mathrm{KrF}$ laser at the ion energy 4 and $10 \mathrm{GeV}$, and with the ArF laser at 0.3 and $3 \mathrm{GeV}$. The transitions and other spectroscopic data mentioned in this paper are obtained from Refs. [33-37]. For some elements (especially for the heavy ones) the databases do not give information on necessary UV transitions, though such transitions with high probability exist for these elements.

One can see from here that one of the restrictions of the technique is the necessity of tuning the ion beam energy to the value determined by the required Doppler shift. This problem can be overcome in part by the use of the oblique laser beam as it follows from Eq. (1). One more

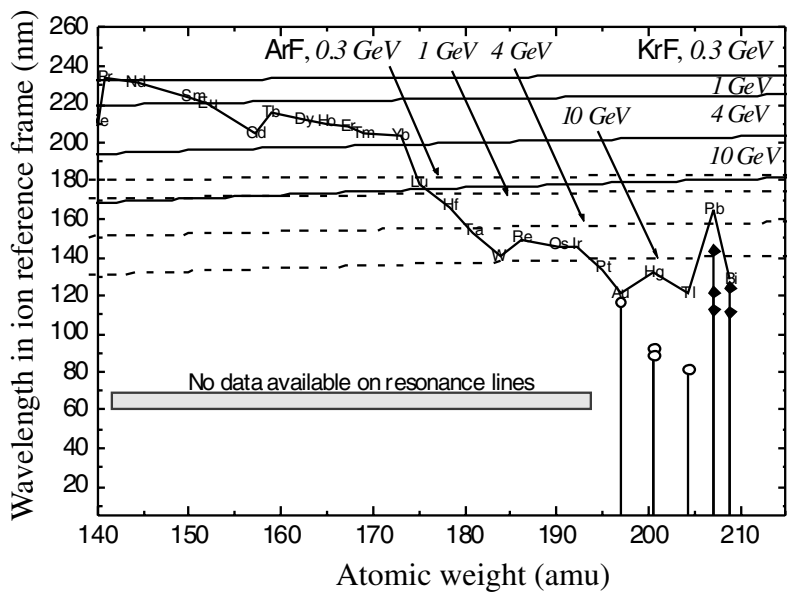

FIG. 4. Wavelengths of the excimer lasers in the moving frame for the singly charged ions of the heavy elements. The legend is the same as in Fig. 2. restriction is the absence for today of the robust hard-UV lasers that are necessary for ionization of the ions with high ionization potentials such as $\mathrm{Ne}^{+}, \mathrm{Na}^{+}, \mathrm{Ar}^{+}, \mathrm{Kr}^{+}$, $\mathrm{Rb}^{+}, \mathrm{Ag}^{+}, \mathrm{Xe}^{+}, \mathrm{Cs}^{+}, \mathrm{Au}^{+}, \mathrm{Hg}^{+}, \mathrm{Tl}^{+}$, and $\mathrm{Bi}^{+}$(see Figs. 2-4). Future development of laser technology will undoubtedly close this gap. The other restrictions of the technique - unfavorable features of the term system for some ions - will be discussed further.

\section{RESONANCE SATURATION OF INTERMEDIATE LEVEL}

For the effective photoionization of the ion beam, the population of the intermediate level has to be as high as possible. The maximum population corresponds to the conditions of the resonance saturation. The saturated population of the upper level $k$ in a system of two levels $k$ and $m$ (in our case the last denotes the ion ground state, $m=1$ ) can be calculated using the semiclassical kinetic equation (for details see, i.e., [38]):

$$
\begin{aligned}
\frac{d n_{k}}{d t}= & n_{m} \int j_{L}(\omega) \sigma_{k m} \omega d \omega \\
& -n_{k} \int\left[j_{L}(\omega) \sigma_{k m}(\omega)+a_{k m}(\omega)\right] d \omega,
\end{aligned}
$$

where $j_{L}(\omega)$ is the spectral density of the photon flux, and

$$
\begin{aligned}
& \sigma_{k m}=\frac{a_{k m} \lambda_{k m}^{2}}{4}, \\
& \sigma_{m k}=\frac{g_{k}}{g_{m}} \cdot \sigma_{k m}
\end{aligned}
$$

are the cross sections of the induced absorption and induced radiation [39]. The partial Einstein coefficient $a_{k m}(\omega)$ for the spontaneous emission is normalized to the integral Einstein coefficient for allowed dipole-dipole transitions $(E 1)$ :

$$
\begin{gathered}
\int a_{k m}(\omega) d \omega=A_{k m}, \\
A_{k m}\left[\mathrm{~s}^{-1}\right]=6.67 \times 10^{13} \frac{g_{k}}{g_{m}} \frac{f_{k m}}{\left(\lambda_{k m}[\mathrm{~nm}]\right)^{2}},
\end{gathered}
$$

where $g_{k}$ and $g_{m}$ are the degrees of degeneracy and $f_{k m}$ is the oscillator strength.

All the equations are written for the atomic system rest frame. By definition, saturation photon flux density $j_{L}^{s}$ corresponds to the equality

$$
j_{L}^{s}(\omega) \sigma_{k m}(\omega)=a_{k m}(\omega) .
$$

Therefore, a transition is saturated if the spectral density is equal to or more than

$$
j_{L}^{s}(\omega)=\frac{a_{k m}}{\sigma_{k m}}=\frac{4}{\lambda^{2}}\left[\frac{1}{\mathrm{~cm}^{2} \mathrm{~s} \mathrm{~s}^{-1}}\right] .
$$

It must be emphasized that $j_{L}^{s}(\omega)$ does not depend on the 
atomic parameters but only on the wavelength of the resonance transition.

Since for $\theta=\pi$ the wavelength converts to $\lambda_{L}=$ $\lambda_{L 0} \sqrt{(1-\beta) /(1+\beta)}$, required saturation spectral density in the ion frame can be written as

$$
j_{L}^{s}(\omega)=\frac{4}{\lambda_{L 0}^{2}}\left(\frac{1+\beta}{1-\beta}\right),
$$

were $\lambda_{L 0}$ is the laser wavelength in the laboratory coordinate system. The beam cross section for $\theta=\pi$ is the same in both systems. Characteristic pulse length (proper time in terms of the relativistic theory) of an excimer laser is about $\tau_{L 0}=25 \mathrm{~ns}$. In the ion reference frame it increases by $\gamma$ times:

$$
\tau_{L}=\gamma \tau_{L 0}
$$

The pulse length $\tau_{L}$ remains practically the same for heavy ions and converts to $\tau_{L} \approx 60 \mathrm{~ns}$ for $10 \mathrm{GeV} \mathrm{He} e^{+}$. The total number of photons is a relativistic invariant

$$
N_{L}^{s}=j_{L}^{s} \Delta \omega_{L} \tau_{L}=\text { inv. }
$$

Substituting here Eqs. (2), (8), and (9), one can find relativistic transformation for photon flux spectral density:

$$
j_{L 0}^{s}(\omega)=\sqrt{\frac{1+\beta}{1-\beta}} \gamma j_{L}^{s}(\omega) .
$$

Thus spectral density of the photon flux, required for saturation of a resonance transition of the moving ion, in the laboratory reference frame is

$$
j^{s}(\omega)=\frac{4}{\lambda_{L 0}^{2}} F(\beta)
$$

where

$$
F(\beta)=\frac{1+\beta}{(1-\beta)^{2}}
$$

Since

$$
j_{\omega} d \omega=-j_{\lambda} d \lambda,
$$

after simple evaluation one comes to the expression for the saturation spectral power density in practical units:

$$
J_{\lambda}^{S}\left[\frac{\mathrm{kW}}{\mathrm{cm}^{2} \mathrm{~nm}}\right]=\frac{1,42 \times 10^{14}}{\lambda_{L 0}^{5}[\mathrm{~nm}]} F(\beta) .
$$

One should emphasize that the saturation intensity does not depend on atomic parameters of the transition and is a function of the wavelength only. For the wavelengths of interest we have

$$
J_{\lambda}^{s}(248.4 \mathrm{~nm})=150 F(\beta)\left[\frac{\mathrm{kW}}{\mathrm{cm}^{2} \mathrm{~nm}}\right] \quad(\text { KrF laser }) ;
$$

$$
J_{\lambda}^{s}(193.3 \mathrm{~nm})=526 F(\beta)\left[\frac{\mathrm{kW}}{\mathrm{cm}^{2} \mathrm{~nm}}\right] \quad \text { (ArF laser). }
$$

For heavy ions the relativistic factor $\gamma$ is close to unity and the function $F(\beta) \approx 1$. Multiplying the required spectral power density (14) and (15) by $\tau_{L 0}$, one can find laser pulse energy necessary for ion beam saturated excitation. For the $\mathrm{KrF}$ laser $\Delta \lambda_{L} \approx 0.4 \mathrm{~nm}$ and for the laser-beam cross section of $1 \mathrm{~cm}$, the laser pulse energy required for saturation of a heavy-ion transition is merely $1.5 \mathrm{~mJ}$. Similar calculation for ArF laser yields about $6 \mathrm{~mJ}$. For relativistic light ions $F(\beta)$ rapidly grows, and for $\left(1 / 4 \gamma^{2}\right)^{-1} \ll 1$ it tends to the limit $F(\beta) \rightarrow 8 \gamma^{4}$. For the lightest (helium) ion at $T=10 \mathrm{GeV}$ the relativistic factor $\gamma \approx 2.5$ and $F(\beta)=278$. Necessary laser energy proportionally grows but still remains very reasonable. This estimation shows that saturated population of the intermediate transition is easily attainable for most ions with conventional excimer lasers.

Since for the excimer lasers $\Delta \lambda_{L 0} / \lambda_{L 0} \equiv \Delta \lambda_{L} / \lambda_{L} \approx$ $2 \times 10^{-3}$ (see Fig. 2 in [40]), the relative Doppler width of the resonance line of the singly charged ion has to be not more than this value. In this case only a fraction of the laser photons equal to $\Delta \lambda_{+D} / \Delta \lambda_{L}$ interacts with the ions. In the opposite case only a fraction of the ions equal to $\Delta \lambda_{L} / \Delta \lambda_{+D}$ can be excited.

\section{PHOTOIONIZATION OF EXCITED SINGLY CHARGED IONS}

Let us assume the bandwidth of laser radiation to be more than the Doppler width of the ion absorption line. For the laser-beam cross section of $1 \mathrm{~cm}^{2}$ the spectral power density $J_{\lambda}$ is about $100 \mathrm{MW} / \mathrm{cm}^{2} \mathrm{~nm}$, which is much higher than the saturation spectral power density. Under such conditions the resonance transition of the singly charged ion is populated to saturation, $n_{k} / n_{m}=$ $g_{k} / g_{m}$, at the very beginning of the laser pulse. In contrast to the process of population of the intermediate level, all laser photons participate in the stripping of the excited ions.

The photoionization rate can be calculated from the equation

$$
-\frac{d n_{+}(t)}{d t}=n_{k}(t) j_{L}(\omega) \Delta \omega_{L} \sigma_{\mathrm{ph}}
$$

where

$$
n_{+}=n_{k}+n_{m}
$$

and $\sigma_{\mathrm{ph}}$ is the photoionization cross section. Since

$$
n_{k}(t)=n_{+}(t) \frac{g_{k}}{g_{k}+g_{m}},
$$

density of the doubly ionized ions grows as

$$
n_{++}(t)=n_{+}(0)\left\{1-\exp \left[\left(\frac{g_{k}}{g_{k}+g_{m}} j_{L}(\omega) \Delta \omega_{L} \sigma_{\mathrm{ph}} t\right)\right]\right\} \text {. }
$$


Thus, the characteristic photoionization time in the moving frame, taking into account relation (10), can be written as

$$
\tau_{\mathrm{ph}}=\frac{\tau_{L} G}{N_{\mathrm{ph}} \sigma_{\mathrm{ph}}}
$$

where $G=\left(g_{k}+g_{m}\right) / g_{k}$ and $N_{\mathrm{ph}}$ is a number of photons in the laser beam. Since $\tau_{\mathrm{ph}}$ and $\tau_{L}$ transform to the laboratory frame in the same manner, one can introduce the photoionization length in the laboratory system:

$$
L_{\mathrm{ph} 0}=\tau_{\mathrm{ph} 0} \beta c=\frac{\tau_{L 0} G \beta c}{N_{\mathrm{ph}} \sigma_{\mathrm{ph}}} .
$$

After passing this distance $63 \%$ of the singly charged ions are stripped to the second ionization state.

The only free parameter in the right part of the equation is the number of photons in the laser beam. Specifying an acceptable photoionization length, we determine a number of photons that is necessary for photoionization:

$$
N_{\mathrm{ph}}=\frac{\tau_{L 0} G \beta c}{L_{\mathrm{ph} 0} \sigma_{\mathrm{ph}}}
$$

The photoionization cross section for an excited ion can be estimated with reasonable precision from the expression [41]

$$
\sigma_{\mathrm{ph}}\left[\mathrm{cm}^{2}\right]=70.9 \times 10^{-18} \cdot \frac{n}{Z^{2}}\left(\frac{I-\hbar \omega_{L}}{\hbar \omega_{L}}\right)^{3},
$$

where $n$ is a principle quantum number of the intermediate state $k$ and $Z$ is a charge number of the ion core. We also have taken into account that $\hbar \omega_{k m}=\hbar \omega_{L}$.

\section{SELECTION OF IONS THAT CAN BE PHOTOIONIZED WITH EXCIMER LASERS}

Lets consider now the role of atomic structure details on the RETPI efficiency. In the previous sections we described a "two-level ion," which does not exist in nature. A real atomic system has two features, which have to be considered.

First, the ground term of a real ion can be a singlet or a multiplet. All initial ions with singlet ground terms can be excited by laser radiation. Resonance wavelengths for such ions are shown in Figs. 2-4 with open circles. The ions with multiplet ground terms are distributed over several levels, hereafter called for simplicity "sublevels." In this case, only one of the sublevels can be excited by laser radiation. The distances between sublevels are mostly more that the width of the generation spectrum.

Second, for heavy ions there usually exist other levels lying below the selected intermediate level. Population of these levels grows with time, and a fraction of the singly charged ions escape photoionization. Obviously, in this case Eq. (17) is not valid and occupation of the intermediate level is described by a system of kinetic equa- tions. Analysis of the system in detail is beyond the scope of this paper and we content ourselves below with simple estimations.

Initial ion distribution over the states in the ion source is usually the Boltzmann one. The extracted beam may contain ions at different states: ground, short-living excited, and metastable. The short-living states decay within about $10 \mathrm{~ns}$. The fraction of the metastable states strongly depends on the kind of the element and for many elements can be rather little. The metastables may decay during ion storage or can be "cleaned off" by excitation to the upper levels (see below).

Figure 5 shows a Grotrian diagram for $\mathrm{Sr}^{+}$. Let us suppose that all the ions are in the singlet ground state. Two $2 s-2 p$ transitions can be used for population of two possible intermediate levels. It is better to use the $4 p^{6} 6 p^{2} P_{1 / 2}^{0}$ intermediate level $(\lambda=179.31 \mathrm{~nm})$, for which the cascade transition system is simpler. Lifetimes for $5 d$ and $5 p$ levels are very low $[42,43]$ in comparison with the laser pulse length $\tau_{L}$, and they cannot practically slow the ionization process down.

The most serious problem is the existence of the metastable $4 d$ levels with lifetimes $\sim 0.4 \mathrm{~s}$ [44], which are populated through $4 d-5 p(\tau=110 \mathrm{~ns})$ and $4 d-6 p(\tau$ is unknown, but probably similar) transitions. Spontaneous radiation lifetimes for these transitions in practical situations must be rather long in comparison with the ionization time (19) in the ion frame; nevertheless this radiation branch may decrease efficiency of ionization.

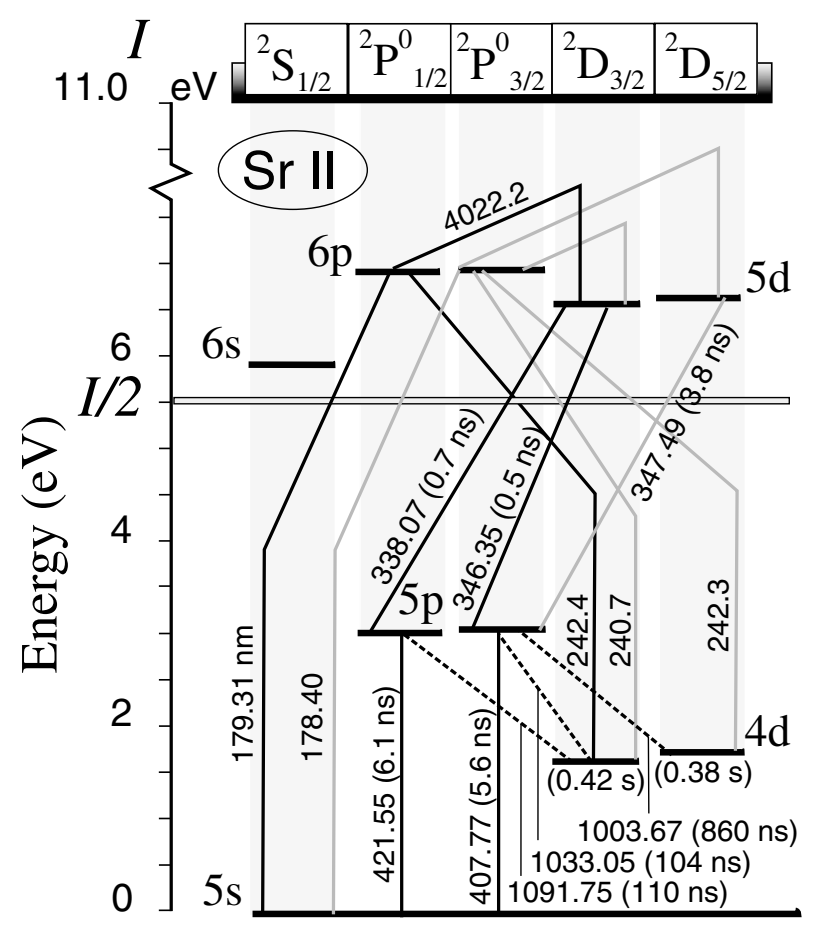

FIG. 5. Grotrian diagram for Sr II. Dark lines denote the transitions involving in the radiative processes for $\lambda_{L}=$ $179.3 \mathrm{~nm}$. Dashed lines denote transitions to metastable states. 
This shortcoming may be obviated by irradiating the ions on the wavelength exciting the metastable atoms up to an allowed transition. In our example it is $\lambda=242.7 \mathrm{~nm}$. It may be a fraction of the same laser radiation propagating through the ion beam under the angle $\theta$ determined by Eq. (1). The absence of data on the Einstein coefficient for many transitions does not enable exact calculation of ionization time and ionization efficiency for many elements, but obviously, the ions with a singlet ground state can be stripped to the next ionization state by means of the RETPI technique.

Consider now the elements with a multiplet ground state. The wavelengths of resonance transitions from sublevels of the multiplet ground state for such ions are shown in Figs. 2-4 with diamonds. For clarity sake we select for analysis (Fig. 6) a tin ion having a relatively simple two-level ground state [45]. Usually the distance in the energy scale between the multiplet components is rather large and only one of the components can be excited by the laser radiation. In this case only a fraction of the ions can participate in RETPI. The value of this fraction depends on the ion prehistory (initial distribution over all the energy levels, spontaneous transition rates, time of ion traveling).

In the general case, one can expect that the ions accelerated to the required velocity may be only in metastable or ground states. We have discussed above how the metastable states can be depleted. The same method can be employed for depletion of sublevels of the ground state. In this case the required angle $\Delta \theta$ between the two laser

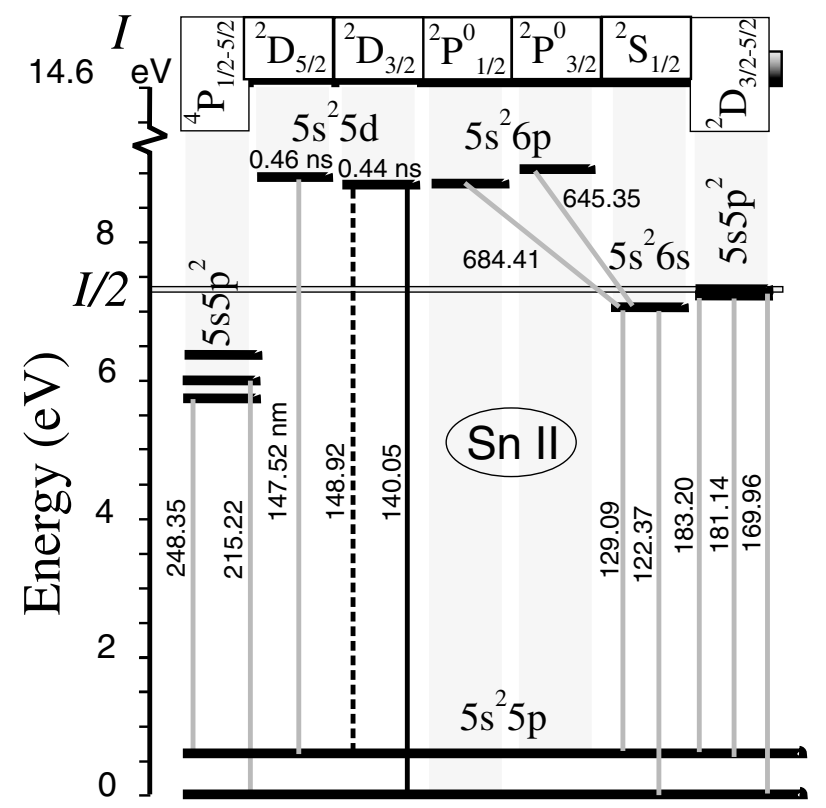

FIG. 6. Grotrian diagram for Sn II. The dark lines denote the transitions involved in the radiative processes for $\lambda_{L}=$ $140.1 \mathrm{~nm}$. The dash line denotes the transition to the longlived upper sublevel that has to be "cleaned." beams, obviously, is very small. The other possible method of cleaning is spontaneous decay. Transitions between the sublevels are forbidden in the electric dipole assumption, but slow magnetic dipole $(M 1)$ or electric dipole (E2) transitions are permissible. The probabilities of these transitions in comparison with the $E 1$ transition for equal $\Delta E$ are $10^{-5}$ and $10^{-8}$, respectively [46]. Since $A_{k m}(M 1) \propto \lambda_{k m}^{3}$ and $A_{k m}(E 2) \propto \lambda_{k m}^{5}$, for small distances between sublevel components the probability of radiative decay of the upper sublevels is very small. Degree of the forbiddenness, however, decreases for heavy elements, and for some experimental configurations spontaneous decay may work.

In the specific case of the Sn II ion it is better to select as the intermediate level the $5 s^{2} 5 d^{2} D_{3 / 2}^{0}$ state. It has no obvious allowed transitions to lower levels (transitions with a simultaneous change of state for two electrons have low probability), besides a very fast transition to the upper level of the ground state (the last means that radiative cleaning of this level is absolutely necessary). The transition $5 s^{2} 5 p^{2} P_{1 / 2}^{0}-5 s 5 p^{2} D_{3 / 2}^{0}$ corresponds to $\lambda=169.96 \mathrm{~nm}$. It is only $0.47 \mathrm{~nm}$ more than it requires for a state to be employed as the intermediate level. Although there is no data on the transition probability, by analogy with Tl II (see, e.g., [47]) it must be about twice less than for the above mentioned $5 p-6 s$ transition. Using two inclined laser beams and increasing a little the ion beam energy, it is easy to design a system employing this transition. These two examples show that for stripping of singly charged ions of a specific element one has to develop an individual optical system with one or several laser beams.

\section{PROTON INJECTION}

Although this paper is devoted to stripping the ions having at least two electrons, application of the RETPI method for production of protons is a subject of special interest because of the wide applicability of proton machines. Electron photodetachment from $\mathrm{H}^{-}$and subsequent ionization of the resulting atom by laser excitation to Rydberg states and high electric field ionization was suggested in a paper by Zelensky et al. [48], which was devoted to the production of a polarizing relativistic proton beam. Yamane [22] suggested modification of this method. The $\mathrm{H}^{-}$ions are neutralized by means of the Lorentz stripping in a magnetic field gradient. The resulting hydrogen atoms are excited by laser radiation to the $3 p$ state and then lose the last electron by stripping in a magnetic field gradient. This method is rather promising, taking into account the fact that the Lorentz stripping of $\mathrm{H}^{-}$is already realized in the Los Alamos proton storage ring [49].

A similar technique for proton injection in the storage ring of the U.S. Spallation Neutron Source (SNS), which alleviated some difficulties inherent to the Yamane 
technique, was described several months ago by Danilov et al. [23]. Both techniques require excitation of Rabi oscillations in the two-level atomic system and synchronizing them with passing the gradient to provide complete Lorentz ionization of hydrogen atoms at the sharply growing magnetic field. This applies very rigid requirements to laser characteristics and to the geometry of the laser-atomic beam interaction. A drawback of the technique is the growth of the proton beam emittance because of the finite stripping length in the strong magnetic field. For example, for the system considered in [22] the additional divergence angle for the protons produced is $1-10 \mathrm{mrad}$ depending on the proton energy $(1-3 \mathrm{GeV})$ and the magnetic field gradient $(3-100 \mathrm{~T} / \mathrm{m})$.

Application of the RETPI technique for ionization of fast atomic hydrogen beams can, probably, be competitive in comparison with the above mentioned stripping methods. An obvious advantage is the absence of additional growing of the beam emittance that inevitably appears for stripping in the magnetic field. The other advantage is the possibility of using a simple and robust excimer laser. For atom stripping one can use a rather long straight interaction region and there is no necessity to excite spatially synchronized Rabi oscillations (see for details [50]). Since the hydrogen atom has a singlet ground state all initial atoms can be excited to the intermediate level. If one employs as the intermediate level $2 p$, but not $3 p$, the metastable state $2 s$ is not populated and there are now atoms stored in this state (see Sec. VI). High-pressure $\mathrm{KrF}$ and $\mathrm{ArF}$ lasers have wide and continuous generation spectrum that allows one to cover completely the Doppler width of the real beams and, consequently, the atoms can be ionized with $100 \%$ efficiency.

The scenario for RETPI proton generation is as follows. Negative hydrogen ions are produced by any conventional method and accelerated to the energy that is required for RETPI [see Eq. (1)]. Then they are transformed into neutral atoms either by Lorentz stripping or by photodetachment:

$$
\mathrm{H}^{-}+\hbar \omega_{1} \rightarrow \mathrm{H}+e^{-} .
$$

Since the cross section of the detachment as a function of wavelength [51] has a wide maximum from 400 to $1200 \mathrm{~nm}$ (FWHM) with $\sigma_{\operatorname{det}}^{\max } \approx 3.5 \times 10^{-17} \mathrm{~cm}^{2}$, the radiation may be produced alternatively with a flashlamp or a laser. The neutrals produced are stripped by excimer laser radiation, whose wavelength is matched to the $1 s-2 p$ transition $(\lambda=121.57 \mathrm{~nm})$ in the atom rest frame:

$$
\mathrm{H}+2 \hbar \omega_{2} \rightarrow \mathrm{H}^{*}+\hbar \omega_{2} \rightarrow \mathrm{H}^{+}+e^{-} .
$$

Resonance saturation of the intermediate level for the $\mathrm{KrF}$ laser can be obtained at the atom energy of about $300 \mathrm{MeV}$ and about $100 \mathrm{MeV}$ for the ArF laser (see Fig. 2). Photoionization cross section for the excited atom is $\sigma_{\mathrm{ph} \mathrm{H}^{*}} \approx 0.6 \times 10^{-18} \mathrm{~cm}^{2}$. These values look to be ac- ceptable for proton injection. Laser energy required for the photoionization will be calculated in the next section. A more detailed analysis of RETPI for hydrogen is out of the scope of this paper and will be done elsewhere.

\section{CONCLUDING REMARKS}

There are many uses for the resonantly enhanced photoionization in the accelerator technique. First of all, it can be used for injection of accelerated singly charged ions into storage rings by stripping them to the next stage of ionization. One promising application of RETPI may be stacking ion beams in some schemes of the heavy-ion inertial fusion and neutron sources. The technique can be realized even with commercially available excimer lasers. It can be also used as a diagnostic method for studying such characteristics as emittance, energy straggling, bunch length, and density of ion beams in accelerators and storage rings.

One more possible application of RETPI is in the production of ions at local points within an accelerating structure. A gas jet coming across the structure is irradiated with one or two laser beams. The first beam populates an intermediate level and the next one photoionizes the excited atoms. Since the ionization potential for the atoms is lower than for the ions, it is possible to ionize the atoms resting in the laboratory frame. About 20 atoms (see Ref. [40]) have resonance transitions, where resonance lines are covered by the generation bands of the excimer lasers. In this case there is no necessity to use the second laser because $\lambda \leq 4 \pi \hbar c / I$. For several elements (iron, tin, and tantalum) the resonance transitions for both the atom and the singly charged ion coincide at the rest frame with the wavelength of the $\mathrm{KrF}$ laser [40]. This coincidence allows obtaining a beam of singly charged ions about half of which is excited to a definite term. This feature can probably also be used, for example, for diagnostics of the accelerating structure recording the fluorescence during the course of acceleration.

In conclusion, I present in Table I the laser energy density

$$
F=N_{\mathrm{ph}} \cdot \hbar \omega_{L 0}
$$

TABLE I. Kinetic energy of ions $T$, for which the resonance transitions coincide with Doppler shifted laser wavelength, and laser energy density providing the ion photoionization length $L_{\text {ph } 0}$ to be equal to $1 \mathrm{~m}$.

\begin{tabular}{cccccc}
\hline \hline & & \multicolumn{2}{c}{ KrF laser } & \multicolumn{2}{c}{ ArF laser } \\
Ion & Transition & $T, \mathrm{Gev}$ & $F_{L 0}, \mathrm{~J} / \mathrm{cm}^{2}$ & $T, \mathrm{Gev}$ & $F_{L 0}, \mathrm{~J} / \mathrm{cm}^{2}$ \\
\hline Sn II & $5 p-5 d$ & $\ldots$ & $\ldots$ & 6 & 1.4 \\
Sr II & $5 s-6 p$ & 3 & 1.3 & 0.3 & 0.54 \\
Ca II & $4 s-5 p$ & 3.5 & 2.4 & 0.5 & 1.2 \\
Mg II & $3 s-4 p$ & 6 & 7.4 & 2.5 & 6.7 \\
He II & $1 s-2 p$ & 11 & 53 & 9 & 67 \\
H I & $1 s-2 p$ & 0.3 & 8.9 & 0.1 & 7.5 \\
\hline \hline
\end{tabular}


which is required to obtain the rest-frame ionization length $L_{\mathrm{ph} 0}$, see Eqs. (20) and (21), equal to $1 \mathrm{~m}$, for several singly charged ions and the hydrogen atom. The laser pulse length is $25 \mathrm{~ns}$. The atomic parameters correspond to the transitions given in the second column of the Table. Practically for all ions we have $\sigma_{\mathrm{ph}} \sim$ $10^{-18}-10^{-19} \mathrm{~cm}^{2}, G \sim 1-2$, and $\beta \sim(0.1-1)$. The necessary photon number per $\mathrm{cm}^{2}$ lies within the limits

$$
N_{\text {ph }} \sim 10^{18}-5 \times 10^{19} \text {. }
$$

The required laser energy density is about $1 \mathrm{~J} / \mathrm{cm}^{2}$ for heavy ions (quite reasonable energy, easily achieved for such lasers) and abruptly grows up to $50 \mathrm{~J} / \mathrm{cm}^{2}$ for the lightest ions. For the particular case of hydrogen atom ionization in the SNS the beam cross section is less than $2 \times 2 \mathrm{~mm}$ [23], and in accordance with Table I the required $\mathrm{KrF}$-laser pulse energy is only $0.3 \mathrm{~J}$.

Thus, an excimer laser with rather moderate parameters can ensure practically complete photoionization of the ion bunch in a straight channel of several meter length during the $25 \mathrm{~ns}$ laser pulse. The estimation above is correct if the total ionization energy is much less than the laser pulse energy. The ionization of $10^{14}$ ions, for example, requires as little as $200 \mu \mathrm{J}$. This means that the excimer laser beam can be, if used with adequate reflectors and optical delays, multiply employed for ionization of many subsequent ion bunches. One of the possible optical systems is described in [23].

\section{ACKNOWLEDGMENTS}

The author is indebted to V. Danilov, G. I. Dimov, I. B. Khriplovich, I. N. Meshkov, A. I. Milstein, K. A. Nasyrov, V.V. Parkhomchuk, and A. M. Shalagin for helpful comments and discussions. This work is based, in part, on the experimental results obtained under a grant from the program "Russian Universities Fundamental Research" on the CATRION setup (Reg. No. 06-06, NSU) supported by the Ministry of Industry, Science and Technology of the Russian Federation.

[1] A. J. Dempster, Phys. Rev. 42, 901 (1932).

[2] L.W. Alvarez, Rev. Sci. Instrum. 22, 705 (1951).

[3] J. Peters, Rev. Sci. Instrum. 71, 1069 (2000).

[4] G. I. Budker and G. I. Dimov, in Proceedings of the International Conference on High Energy Accelerators, Dubna, 1963 (Atomizdat, Moscow, 1964), p. 993.

[5] G. I. Budker et al., At. Energ. 19, 507 (1965) [Sov. J. At. Energy].

[6] G. I. Dimov, INP Report No. 304, Novosibirsk, 1969 (in Russian).

[7] G. I. Dimov, Rev. Sci. Instrum. 67, 3393 (1996).

[8] R. Maier et al., Nucl. Phys. A626, 395c (1997).

[9] L. Hermansson and D. Reistad, Nucl. Instrum. Methods Phys. Res., Sect. A 441, 140 (2000).
[10] A. D. Kovalenko, in Proceedings of the EPAC 2000, Vienna, Austria (CERN, Geneva, 2000), Vol. 1, p. 554.

[11] L. Dahl, W. Barth, and S. Yaramishev, in Proceedings of the XXI International Linac Conference, Gyeongju, Korea, 2002 (Pohang Accelerator Laboratory, Pohang, Korea, 2003).

[12] E. Jaeschke et al., in Proceedings of the European Particle Accelerator Conference, Rome, 1988 (World Scientific, Singapore, 1989), Vol. 1, p. 365.

[13] B. Sharkov et al., Nucl. Instrum. Methods Phys. Res., Sect. A 415, 20 (1998).

[14] P. Zenkevich et al., Nucl. Instrum. Methods Phys. Res., Sect. A 464, 616 (2001).

[15] I. Hofmann, Nucl. Instrum. Methods Phys. Res., Sect. A 278, 271 (1989).

[16] B. Yu. Sharkov, in Proceedings of the 14th International Symposium on Heavy Ion Fusion, Moscow, 2002, Abstracts (Institute of Experimental and Theoretical Physics, Moscow, 2002), p. 10.

[17] I. Hofmann, Nucl. Instrum. Methods Phys. Res., Sect. A 464, 24 (2001).

[18] D. H. H. Hoffmann, in Proceedings of the 14th International Symposium on Heavy Ion Fusion, Moscow, 2002, Abstracts (Ref. [16]), p. 8.

[19] G. B. Logan, in Proceedings of the 14th International Symposium on Heavy Ion Fusion, Moscow, 2002, Abstracts (Ref. [16]), p. 9.

[20] D. Dinev, Nucl. Instrum. Methods Phys. Res., Sect. A 479, 254 (2002).

[21] C. Rubbia, Nucl. Instrum. Methods Phys. Res., Sect. A 278, 253 (1989).

[22] I. Yamane, Phys. Rev. ST Accel. Beams 1, 053501 (1998).

[23] V. Danilov et al., Phys. Rev. ST Accel. Beams 6, 053501 (2003).

[24] A. A. Doroshkin et al., in Proceedings of the 14th International Symposium on Heavy Ion Fusion, Moscow, 2002, Abstracts (Ref. [16]), p. 58.

[25] B. A. Knyazev, in Proceedings of the 10th International Conference on Ion Sources, Dubna, Russia, 2003, Book of Abstracts (JINR, Dubna, 2003), p. 210; Rev. Sci. Instrum. (to be published).

[26] R. Arnold, R. Burke, Y. Cho, and R. Cutler, IEEE Trans. Nucl. Sci. 24, 1428 (1977).

[27] I. Hofmann, Laser Part. Beams 8, 527 (1990).

[28] J. B. West, J. Phys. B 34, R45 (2001).

[29] M. V. Yurkov et al., Nucl. Instrum. Methods Phys. Res., Sect. A 483, 51 (2002).

[30] Y. R. Shen, The Principles of Nonlinear Optics (John Wiley, New York, 1984).

[31] V.S. Letokhov, Laser Photoionization Spectroscopy (Nauka, Moscow, 1987) (in Russian).

[32] D. Basting and U. Stamm, Z. Phys. Chem. 215, 1575 (2001).

[33] A.S. Yatsenko, Grotrian Diagrams of Singly-Charged Ions (Nauka, Novosibirsk, 1996) (in Russian).

[34] Ch. E. Moore, Atomic Energy Levels, NBS Circular No. 467 (U.S. GPO, Washington, DC, 1949), Vols. I-III.

[35] S. Bashkin and J. O. Stoner, Atomic Energy Levels and Grotrian Diagrams (North-Holland, Amsterdam, 1975), Vols. I-II. 
[36] National Institute of Standards and Technology Atomic Spectroscopic Database, http://physics.nist.gov// PhysRefData/contents.html; P. L. Smith, C. Heise, J. R. Esmond, and R. L. Kurucz, Atomic Spectral Line Database, http://cfa-www.harvard.edu/amdata/ampdata/ kurucz23/sekur.html

[37] Ch. H. Corliss and W. R. Bozman, Experimental Transition Probabilities for Spectral Lines of Seventy Elements, Natl. Bur. Stand. No. 53 (U.S. GPO, Washington, DC, 1963).

[38] A. Maitland and M. H. Dunn, Laser Physics (NorthHolland, Amsterdam, 1969).

[39] V. P. Krainov and B. M. Smirnov, Radiation Processes in Atomic Physics (Vysshaya Shkola, Moscow, 1983) (in Russian).

[40] B. A. Knyazev, Nucl. Instrum. Methods Phys. Res., Sect. A 415, 525 (1998).

[41] Ya. B. Zeldovich and Yu. P. Raiser, Physics of Shock Waves and High-Temperature Hydrodynamic Phenomena (Nauka, Moscow, 1966) (in Russian).

[42] T. Brage, G. M. Wahlgren, S. G. Johansson, D. S. Leckrone, and C. R. Proffitt, Astrophys. J. 496, 1051 (1998).
[43] A. L. Osherovich, Ya. F. Verolainen, and S. A. Pulkin, Opt. Sectrosc. 46, 253 (1979) (in Russian).

[44] E. Biémont, J. Lidberg, S. Mannervik, L.-O. Norlin, P. Royen, A. Schmitt, W. Shi, and X. Tordoir, Eur. Phys. J. D 11, 355 (2000).

[45] R. M. Schectman, S. Cheng, L. J. Curtis, S. R. Federman, M. C. Fritts, and R. E. Irving, Astrophys. J. 542, 400 (2000).

[46] I. I. Cobelman, Introduction to Theory of Atomic Spectra (Fizmatgiz, Moscow, 1963), p. 388.

[47] I. B. Khriplovich, Parity Nonconservation in Atomic Phenomena (Gordon and Breach, New York, 1991).

[48] A. N. Zelensky, S. A. Kokhanovsky, V. M. Lobashev, N. M. Sobolevskiy, and E. A. Volferts, Nucl. Instrum. Methods Phys. Res., Sect. A 227, 429 (1984).

[49] D. Fitzgerald, in Proceedings of the 6th ICFA Beam Dynamics Mini-Workshop on Injection and Extraction in High Intensity Proton Machine, 1999, Abingdon.

[50] D. F. Walls and G. J. Milburn, Quantum Optics (Springer, New York, 1994).

[51] H. Massey, Negative Ions (Cambridge University Press, Cambridge, UK, 1976). 